P154 (continued)

Objective: To seek feedback from intended users regarding short videos designed to educate low-income parents about evidence-based recommendations for feeding young children, and guidance for development of future videos.

Study Design, Setting, Participants, Intervention: Participants $(\mathrm{n}=18)$ included parents (94\% female; $89 \%$ Hispanic) of children enrolled in Head Start or Early Head Start in Hays and Caldwell counties in south central Texas. Participants viewed eight 90 -second, bilingual videos instructing on evidence-based recommendations for feeding practices, and completed scorecards that posed questions, with 5-point Likert scale responses, about perceptions of the videos with respect to quality, entertainment, pedagogical efficacy, intent to use the educational content, and overall reaction. Additionally, semi-structured interviews, conducted in English or Spanish, asked about perceived benefit of videos and requested suggestions for design of future videos.

Outcome Measures and Analysis: Frequencies of responses to scorecard questions were calculated. Interviews were transcribed, with Spanish interviews first translated to English and back-translated. A three-coder model was used to code interviews for emergent themes.

Results: The majority of participants rated video quality as positive, and were enthusiastic about learning from videos. Participants expressed intent to use comparable videos to obtain nutrition and food-related information.

Conclusion and Implications: Brief instructional videos show promise as an effective method for disseminating feeding practice recommendations for young children to parents. As bilingual videos were preferred by Spanishspeaking and bilingual parents, implementing a bilingual design for videos for us by Head Start parents in Texas may be warranted.

Funding: Caminitos Research Initiative, Texas State University.

\section{P155 What's for Dinner? Analyzing the Dinner Meal of Parent-Preschool Child Dyads Using Food Photography}

Morgan McCloskey, MSPH, Colorado State University; Susan L. Johnson, PhD, University of Colorado, Anschutz Medical Campus; Traci A. Bekelman, PhD; Corby K. Martin, PhD, Pennington Biomedical Center; Laura L. Bellows, PhD, MPH, RD, laura.bellows@colostate.edu, Colorado State University, Department FSHN, 1571 Campus Delivery, Fort Collins, CO 80523-1571

Background (Background, Rationale, Prior Research, and/or Theory): Despite the substantial contribution of dinner meals to preschoolers' nutrient intake, limited data on dinner meals exists from families with young children. These data are important since the home environment influences eating behaviors and meals in the home are a potential intervention target.
Objective: To utilize the Remote Food Photography Method $^{\circledR}$ (RFPM) to characterize typical dinner meals of parent-preschooler dyads.

Study Design, Setting, Participants, Intervention: Participants were recruited from five Head Start/preschool centers in rural, eastern Colorado. Participants attended a 1.5-hour training on the RFPM and SmartIntake app and were provided iPads to take photos of the dinner meal for themselves and their child for seven consecutive dinner meals. Data were wirelessly transmitted in near real-time for review of data quality and completeness.

Outcome Measures and Analysis: Food intake data from the dyads were analyzed to quantify meal timing and concordance in parent-child meal composition and meal quality, as assessed by comparison to a variety of indices, including Child and Adult Food Care Program (CACFP) guidelines and the Healthy Meal Index (HMI), a validated tool for assessing the quality of meals served to preschoolers.

Results: Participants were mothers (97\%), 45\% Hispanic, and $77 \%$ low-income. Mothers ate similar foods during $74 \%$ of meals and at the same time during $93 \%$ of meals as their preschooler. Few meals met CACFP guidelines for milk (14\%) or whole grains (17\%). The mean HMI score was $66.7 \pm 8.9$ (possible score $=105$ ). Protein and dairy (mostly cheese) were the highest scoring components, with whole grains, fruit, vegetable variety and added saturated fat being the lowest scoring components.

Conclusions and Implications: Mothers and preschoolers in this sample ate similar foods together at the same time for dinner, providing an opportunity for role modeling of healthy eating behaviors. Future intervention targets may include serving milk, more variety of whole grains, nutrient-rich vegetables, and fewer foods high in saturated fat.

Funding: NIFA.

\section{Nutrition Education Program Design Implementation and Evaluation}

\section{P156 Assessment of Policy, Systems and Environmental (PSE) Change Strategies Delivered Through Maine SNAP-Ed}

Brent Walker, MS, RD, brent.walker@altarum.org, Altarum, 4 Milk Street, Suite 303, Portland, ME 04101; Brenda Wolford, MS, RD; Rebecca Hofer, MPH; Patricia Dushuttle, MA, State of Maine Department of Health and Human Services, Office for Family Independence

Objective: To assess the implementation of PSE change strategies through Maine's Supplemental Nutrition Assistance Program-Education (SNAP-Ed).

Target Audience: Maine SNAP-Ed educators, administrators and partners. 